(C) 2019. This manuscript version is made available under the CC-BY-NC-ND 4.0 license http:/creativecommons.org/licenses/by-nc-nd/4.0/

\title{
MMP-3 and MMP-8 in Rat Mandibular Condylar Cartilage Associated with Dietary Loading, Estrogen Level, and Aging
}

${\text { Jia } \mathrm{Yu}^{1 *} \text {, Eerika Mursu}{ }^{1} \text {, Matleena Typpo }{ }^{1}, \text { Sakari Laaksonen}^{2} \text {, Hanna-Marja Voipioº }}^{2}$, Paula Pesonen ${ }^{3}$, Aune Raustia ${ }^{1,4}$, Pertti Pirttiniemi ${ }^{1,4}$

1Research Unit of Oral Health Sciences, University of Oulu, Oulu, Finland 2Laboratory Animal Centre, University of Oulu, Oulu, Finland 3Northern Finland Birth Cohort Studies, University of Oulu, Oulu, Finland 4Medical Research Center, Oulu University Hospital, Oulu, Finland

\begin{abstract}
Objectives: The structure of the mandibular condylar cartilage (MCC) is regulated by dynamic and multifactorial processes. The aim of this study was to examine the effects of altered dietary loading, estrogen level, and aging on the structure of the condylar cartilage and the expressions of matrix metalloproteinase (MMP) -3 and MMP-8 of rat MCC.

Methods: In this study, Crl:CD (SD) female rats were randomly divided into 3 groups according to dietary hardness: hard diet (diet board), normal diet (pellet), and soft diet (powder). In each group, the rats were further divided into 2 subgroups by ovariectomy at the age of 7 weeks. The rats were sacrificed at 5- and 14-month-old. Histomorphometric analysis of the MCC thickness was performed after toluidine blue staining. Immunochemical staining was done for MMP-3 and MMP-8. A linear mixed model was used to assess the effects of dietary loading, estrogen level, and aging. Results: Increased dietary loading was the main factor to increase the MMP-3 expression and the anterior and central thickness of the MCC. Lack of estrogen was the main factor associated with decreased MMP-8. Aging was associated with the thickness changes of the whole condylar cartilage and the reduced expression of MMP-8.

Conclusion: The condylar cartilage structure and metabolism of the female rats are
\end{abstract}


sensitive to dietary loading changes, estrogen level as well as aging. The proper balance of these factors seems to be essential for the maintenance of the condylar cartilage.

Keywords: temporomandibular condyle; matrix metalloproteinases; mastication function; gender

\section{Introduction}

Mandibular condylar cartilage (MCC), a primary part and growth site of the temporomandibular joint (TMJ), consists of chondrocytes and extracellular matrix (ECM) that contains collagens, proteoglycans, and water (Embree et al., 2010). Matrix metalloproteinases (MMPs) are major players in ECM developmental and repair processes at both physiological and pathological levels (Murphy et al., 2002). An imbalance between the synthesis and degradation of ECM is a characteristic of degenerative changes in the MCC (Learreta, Bono, \& Durst, 2011).

MCC is able to remodel its structure in response to loading. Normal loading promotes the metabolism of the chondrocytes by increasing the proteoglycan content and cartilage thickness and by decreasing proteoglycan extractability (Betti et al., 2018; Enomoto et al., 2010). Reduced loading induced by small-size diet or botox injection into the masseter causes decreased proliferation, differentiation, and maturation of chondrocytes (Dutra et al., 2018; Liu et al., 2014). Excessive loading leads to degenerative changes in articular cartilage and subchondral bone (Tanaka, Detamore, \& Mercuri, 2008; Wang, Zhang, Gan, \& Zhou, 2015).

Controversy remains about the role of estrogen in the etiology of temporomandibular disorders (TMD) that are a group of problems affecting TMJ, masticatory muscles and other related structures (Ohrbach \& Dworkin, 2016; Perez et al., 2013). TMD predominantly afflict women, and the women-to-men ratio of patients treated for TMD is about 3:1 (Manfredini et al., 2011). The prevalence of TMD increases after puberty, peaks during the reproductive years, and decreases after menopause (Warren \& Fried, 2001). Previous studies have discovered the existence of estrogen receptors in human and animal TMJ (Aufdemorte et al., 1986; Henry, Tull, Whittum-Hudson, \& Wolford, 2008). Hence, it is suspected that sex hormones, particularly estrogen, contributes to 
the incidence of TMD (Kamiya et al., 2013; Traub \& Ji, 2013).

Aging is one of the most dominant factors associated with cartilage degeneration (Mähler et al., 2014). Both the degree and frequency of cartilage breakdown seem to increase with aging (Kuroda et al., 2009). Aging causes disabilities of the chondrocytes and the intracellular matrix to maintain cartilage integrity and homeostasis (Loeser, 2009). It has also been reported that aging is accompanied by a declining number of chondrocytes, lower cartilage turnover, and increased cartilage stiffness (Jorgensen, Kjaer, \& Heinemeier, 2017).

MMP-3 degrades most ECM components, such as aggrecan, type II and type X collagen, ultimately causing cartilage and bone loss (Williams, Feldmann, \& Maini, 2000). As a predictor of joint destruction, MMP-3 also activates procollagenases and seems to play a pivotal role in the initiation of collagen damage (Green et al., 2003; van Meurs et al., 1999). MMP-8 is able to cleave native triple helical collagens, such as type I, type II and type III (Murphy et al., 2002). Studies on humans and animals indicate that MMP3 and MMP-8 are involved in different stages of TMJ pathogenesis (Jiao et al., 2016; Tiilikainen, Pirttiniemi, Kainulainen, Pernu, \& Raustia, 2005a).

The aim of this study was to examine the effects of altered dietary loading, estrogen level, and aging on the structure of the condylar cartilage and the expressions of MMP3 and MMP-8 of rat MCC. Our hypothesis was that altered dietary loading, estrogen level, and aging all play a significant role in the structure and composition of the cartilage.

\section{Methods}

\section{Animals and Husbandry}

Outbred specific pathogen-free Crl:CD (SD) female rats born in the Laboratory Animal Centre, University of Oulu, Finland, were used. At the beginning of the study, they were 8 weeks old and the weights were $221 \pm 20 \mathrm{~g}$ (mean \pm SD). Microbiological health monitoring (serum antibodies, bacterial culture, PCR and direct microscopy) of the animal unit rats was carried out annually for all pathogens listed in the Federation of European laboratory Animal Science Associations recommendations (Mähler et al., 
2014). All results for 18 months before and during the experiment were negative, apart from S.aureus detected in 2015. The animal care and experimental procedures were in line with the Finnish legislation and EU Directive 2010/63/EU. The animal experiment was approved by the National Animal Experiment Committee of Finland.

\section{Diet Board}

In the diet board (Tapvei, Kiili, Estonia), food pellets were tightly fitted into grooves in $2.7 \times 14 \times 36 \mathrm{~cm}$ aspen boards. Two boards with altogether 40 vertical grooves (diameter $12 \mathrm{~mm}, 6 \mathrm{~mm}$ wide gap) were joined in the middle to form a cross-shaped wall structure in the cage (Laaksonen et al., 2017). The rats have to gnaw the wooden boards to reach the food. Therefore, increased TMJ loading was due to gnawing the wooden boards. The boards were changed once a week and turned upside down on the fourth day to keep the eating workload more constant.

\section{Animal model}

Before the experiment, 96 rats were randomly divided into 6 equal groups according to dietary hardness and estrogen status (ovariectomized (OVX) or intact (non-OVX)): OVX rats on the diet board, and non-OVX rats on the diet board, OVX rats on pellet, non-OVX rats on pellet, OVX rats on powder, non-OVX rats on powder. Four birth cohorts of 24 rats were comprised of four litters each, with littermates allocated randomly into different study groups. Before ovariectomy, all rats received pellets as food. The OVX-group rats were operated at 7 weeks of age and the feeding experiment started one week later. The study length was 100 days for two cohorts and 360 days for the other two, so rats were either 5- or 14- month-old at the end. One rat in the 14month-old non-OVX diet board died due to an eye problem; two rats died in 14-monthold non-OVX powder group due to either breast or kidney tumor (Table 1). All rats of the same group in a cohort shared the same cage. Every cohort was housed in its own cage rack.

\section{Tissue Preparation}

At the end of the experiment, rats were pre-anesthetized with isoflurane and then euthanized by $100 \%$ carbon dioxide. The craniums were fixed in $4 \%$ formalin for 7 days, and decalcified with EDTA (pH 7.4) at $37^{\circ} \mathrm{C}$ for 6 weeks. After decalcification, 
the craniums were divided sagittally into left and right TMJs, and then embedded in paraffin. The right TMJs were used in the present experiment and were cut into serial $5-\mu \mathrm{m}$ thick sagittal sections. The most central sagittal sections of the condyle confirmed by toluidine blue staining were selected for further analyses and staining.

\section{Histomorphometric Analysis}

The most central sections stained by toluidine blue from each condyle were examined with a microscope at $2.5 \mathrm{x}$ (Leica, Leitz-Wezlar, Germany). The articular surface was divided equally into anterior, central and posterior parts by using Rhinoceros 5 software (Robert McNeel \& Association, USA). The measurement point was the midpoint of the anterior, central and posterior part (Fig. 1). The thickness was identified from the fibrous to erosion zone in the subchondral bone and was measured at the three points.

\section{Immunohistochemistry}

Deparaffinized sections were treated with $1 \% \mathrm{H}_{2} \mathrm{O}_{2}$ for $30 \mathrm{~min}$ at room temperature to quench endogenous peroxidase activities, and treated with $0.4 \%$ pepsin for 45 min at $37^{\circ} \mathrm{C}$. The sections were then pre-incubated with primary antibodies: 1:50 Goat polyclonal anti-MMP-3 (Santa Cruz Biotechnology, Inc., California, USA) and 1:3000 rabbit polyclonal anti-MMP-8 (Chemicon International, Inc., California, USA).

MMP-8 specimens were incubated with the primary antibody for $60 \mathrm{~min}$ at room temperature. Afterwards, MMP-8 sections were incubated with Dako's HRP rabbit solution (Dako Denmark A/S, Denmark) for 30 min.

After inserting the primary antibody, MMP-3 specimens were placed at $37^{\circ} \mathrm{C}$ for 30 min, and in a fridge at $4^{\circ} \mathrm{C}$ overnight. The next day, MMP-3 sections were applied with Goat Probe (Biocare Medical, LLC, California, USA) and Goat-on-Rodent HRPPolymer for $15 \mathrm{~min}$, respectively.

Negative controls were prepared the same way without the primary antibody. Finally, all the sections were processed with diaminobenzidine (DAB) as the chromogen (3 min for MMP-3, 5 min for MMP-8) and counterstained with Mayer's hematoxylin.

\section{Immunohistochemical Analysis}

The MCC was exhibited with a microscope at 10x (Leica, Leitz-Wezlar, Germany). Then, the images from the same MCC were merged into one whole condylar head 
image with Adobe Photoshop (Adobe Systems, Inc. USA). The accurate MCC was separated with Image J (Maryland, USA) selection tools and the area of the MCC was measured. Immunostaining against antibody was then visualized by applying a color filter and the positive stained area was measured. All the measurements were performed blindly by one investigator. The same threshold was used for each antibody. The analysis registered the stained area divided by the whole area of the MCC.

\section{Statistical Analysis}

The type III tests of fixed effects for thickness, MMP-3 and MMP-8 was made by the linear mixed model with the independent variables being dietary loading, estrogen level, aging, and the two-way interaction terms. The comparisons of groups for the ANOVA analysis were planned on the grounds of these models.

The variations between different classes were examined with the repeated measures ANOVA model. The Tukey-Kramer's multiple comparison test was used as a post hoc test. In MMP-3 and MMP-8 models, the sections from the same condyle were used as a repeated measure. In thickness models, the three measure points of the anterior, central, and posterior parts were used as a repeated measure. Intraclass correlation coefficients (ICCs) were 0.944 for MMP-3 and 0.992 for MMP-8. $P<0.05$ was considered statistically significant. The statistical analyses were conducted using SPSS 22 (IBM, New York, USA) and SAS Enterprise Guide 5.1 (SAS Institute Inc., North Carolina, USA).

\section{Results}

\section{Histomorphometric analysis}

\section{Anterior thickness of MCC}

Loading and aging were the main factors affecting the anterior thickness of MCC (Table 2). In both estrogen groups and in both time points, the anterior thickness was higher in the pellet rats than in the powder rats (Fig. 2B). In the OVX 14-month-old groups, the anterior thickness was higher it the diet board rats than in the powder rats $(p<0.01$, Fig. 2B). In both estrogen diet board and pellet groups, there was a tendency that the anterior thickness increased in the 14-month-old compared to the 5-month-old rats (Fig. 2B). 


\section{Central thickness of MCC}

Loading and aging were the main factors affecting the central thickness of MCC (Table 2). In both estrogen groups and in both time points, the central thickness was significantly higher in the diet board rats than in the powder rats (Fig. 2). In both estrogen 5-month old groups, the central thickness of diet board rats was higher than the pellet rats $(p<0.01$, Fig. 2). In the non-OVX diet board groups, the central thickness was significantly higher in the 5-month-old rats than in the 14-month-old rats $(p<0.01$, Fig. 2).

\section{Posterior thickness of the MCC}

Aging was the main factor to affect the posterior thickness of MCC (Table 2). In the non-OVX pellet groups, the 14-month-old rats had a significantly higher posterior cartilage layer than the 5-month-old rats ( $p<0.05$, Fig. 2 B).

\section{Immunohistochemical Analysis}

\section{Expression of MMP-3}

Loading was the main factor that affected the expression of MMP-3 (Table 2). In the 14-month-old OVX groups, the expression of MMP-3 in the diet board rats was significantly higher than in the powder rats $(p<0.05$, Fig. 3$)$.

\section{Expression of MMP-8}

Aging and estrogen level were the main factors that affected the expression of MMP-8 (Table 2). In all dietary OVX groups, the expression of MMP-8 was significantly higher in the 5-month-old rats than in the 14-month-old rats (Fig. 4). In the non-OVX diet board and powder groups, the 5-month-old rats showed higher expression of MMP-8 compared to the 14-month-old rats (Fig. 4). In the 14-month-old powder groups, the expression of MMP-8 was significantly lower in the OVX rats than in the non-OVX rats ( $p<0.05$, Fig. 4).

\section{Discussion}

The present results showed that aging affected the thickness changes of the whole condylar cartilage. The anterior and posterior thickness of the MCC increased with aging, whereas the central thickness was found to be decreased. The articular surface 
of the mandibular condyle is covered by fibrocartilage, and the structure is different from the articular surfaces of other joints that are composed of hyaline cartilage (Embree et al., 2010). The thickness of the cartilage has been shown to be dependent on the maturation, aging, health status and loading of the joint (Mirahmadi, Koolstra, Lobbezoo, van Lenthe, \& Everts, 2017). Vascular layers have been constantly found to exist at the anterior and posterior parts of the condyles (Rusu, Pop, Leonardi, Motoc, \& Jianu, 2011). Concurrently, it has been reported that endochondral ossification occurs more actively in the posterior part (Kufley, Scott, \& Ramirez-Yanez, 2017). The present results give more evidence that the anterior and posterior parts are growing sites, even though the anterior part is also impacted by loading due to the direct contact with the TMJ disc during mastication (Nickel, McLachlan, \& Smith, 1988; Pirttiniemi, Kantomaa, \& Tuominen, 1993). Previous studies have shown that reduced loading caused by a soft diet leads to a thinner cartilage (Orajarvi, Thesleff, Hartikainen, Raustia, \& Pirttiniemi, 2015). In this study, the results showed similarly that reduced dietary loading decreases the thickness of the anterior and central parts, but not the posterior part. Based on our findings, it could be speculated that the central part is agerelated and also highly influenced by loading, indicating it plays a vital role in the growth of the MCC by withstanding the forces caused by mastication. The present results show that estrogen level does not change the thickness of the MMC in these 5and 14-month-old groups.

In this study, the higher expression of MMP-3 was linked to the heavy masticatory loading. MMPs are a family of proteases that are involved in connective tissue remodeling, angiogenesis and bone growth (Schultz \& Wysocki, 2009). Normally, MMPs are maintained at a constant low level in the tissue and may be upregulated by inflammatory cytokines, hormones, growth factors, and physical factors (JablonskaTrypuc, Matejczyk, \& Rosochacki, 2016). MMP-2, MMP-9, and MMP-13 have been shown to be significantly overexpressed in osteoarthritic cartilage (Ho et al., 2005; Jackson, Moradi, Smith, Jackson, \& Little, 2014). Elevated expression of MMP-3 has been observed in human osteoarthritic TMJ and synovial membrane (Sun et al., 2014; Tiilikainen, Pirttiniemi, Kainulainen, Pernu, \& Raustia, 2005b). In an earlier 
experiment, higher expression of MMP-3 was found in the rat TMJ of the increased loading group (Pirttiniemi, Kantomaa, \& Sorsa, 2004). These results indicate that increased dietary loading leads to MCC catabolism or degeneration by increasing MMP-3 expression.

It has been shown that MMP-8 is involved in the breakdown of ECM and promotes bone tissue degradation (Chen et al., 2017). In the present study, increased expression of MMP-8 was observed in the 5-month-old group compared to the 14-month-old group. This phenomenon may be due to the capability of MMP-8 to develop and remodel tissue via breakdown of ECM, indicating that MMP-8 may be a sign of active condylar cartilage turnover and also act actively during the MCC growth period (Orajarvi et al., 2015). This effect is consistent with the previous study that MMP-8 deficiency has been shown to be associated with the aggravation of arthritis, suggesting that MMP-8 may play a protective role (Garcia et al., 2010; Gutierrez-Fernandez et al., 2007). It is interesting that in the present study, a higher-level expression of MMP-8 was also seen in the non-OVX groups when estrogen was present. Estrogen seems to have a dual action on the cartilage. It is believed that estrogen has a protective function on joint homeostasis by reducing MMP-2 and MMP-9 and decreasing collagen type II degradation (Nielsen, Christiansen, Stolina, \& Karsdal, 2008). On the other hand, estrogen has been found to aggravate TMJ disease (Kou et al., 2011; Ye et al., 2018). Altogether, these results indicated that aging combined with lack of estrogen decrease the activity of MMP-8, which probably results in less active condylar cartilage turnover. In summary, our findings show that the condylar cartilage structure and metabolism of the female rats are sensitive to altered dietary loading, estrogen level as well as aging. The thickness of the central part of MCC is increased by loading and reduced by aging. The anterior thickness is increased by both loading and aging, while the posterior part only increases in thickness with aging. The MMP-3 expression in MCC is upregulated by increased loading, and decreased expression of MMP-8 is associated with aging and low estrogen level. The proper balance of loading, estrogen level, and aging seems to be essential for the maintenance of the condylar cartilage. 


\section{Conflict of interest}

None

\section{Acknowledgements}

This study was supported by the Chinese Scholarship Council fellowship (201608450067).

\section{References}

Aufdemorte, T. B., Van Sickels, J. E., Dolwick, M. F., Sheridan, P. J., Holt, G. R., Aragon, S. B., \& Gates, G. A. (1986). Estrogen receptors in the temporomandibular joint of the baboon (Papio cynocephalus): an autoradiographic study. Oral Surgery, Oral Medicine, Oral Pathology, 61(4), 307-314.

Betti, B. F., Everts, V., Ket, J. C. F., Tabeian, H., Bakker, A. D., Langenbach, G. E., \& Lobbezoo, F. (2018). Effect of mechanical loading on the metabolic activity of cells in the temporomandibular joint: a systematic review. Clinical Oral Investigations, 22(1), 57-67.

Chen, J., Liu, W., Cao, Y., Zhang, X., Guo, Y., Zhu, Y., . . Wang, J. (2017). MMP-3 and MMP-8 singlenucleotide polymorphisms are related to alcohol-induced osteonecrosis of the femoral head in Chinese males. Oncotarget, 8(15), 25177-25188.

Dutra, E. H., O'Brien, M. H., Logan, C., Tadinada, A., Nanda, R., \& Yadav, S. (2018). Loading of the Condylar Cartilage Can Rescue the Effects of Botox on TMJ. Calcified Tissue International, 103(1), 71-79.

Embree, M. C., Kilts, T. M., Ono, M., Inkson, C. A., Syed-Picard, F., Karsdal, M. A., .. . Young, M. F. (2010). Biglycan and fibromodulin have essential roles in regulating chondrogenesis and extracellular matrix turnover in temporomandibular joint osteoarthritis. American Journal of Pathology, 176(2), 812-826.

Enomoto, A., Watahiki, J., Yamaguchi, T., Irie, T., Tachikawa, T., \& Maki, K. (2010). Effects of mastication on mandibular growth evaluated by microcomputed tomography. European Journal of Orthodontics, 32(1), 66-70.

Garcia, S., Forteza, J., Lopez-Otin, C., Gomez-Reino, J. J., Gonzalez, A., \& Conde, C. (2010). Matrix metalloproteinase- 8 deficiency increases joint inflammation and bone erosion in the $\mathrm{K} / \mathrm{BxN}$ serumtransfer arthritis model. Arthritis Res Ther, 12(6), R224.

Green, M. J., Gough, A. K., Devlin, J., Smith, J., Astin, P., Taylor, D., \& Emery, P. (2003). Serum MMP3 and MMP-1 and progression of joint damage in early rheumatoid arthritis. Rheumatology, 42(1), 83-88.

Gutierrez-Fernandez, A., Inada, M., Balbin, M., Fueyo, A., Pitiot, A. S., Astudillo, A., . . Puente, X. S. (2007). Increased inflammation delays wound healing in mice deficient in collagenase-2 (MMP-8). FASEB Journal, 21(10), 2580-2591.

Henry, C. H., Tull, G. T., Whittum-Hudson, J. A., \& Wolford, L. M. (2008). Analysis of estrogen binding sites of the posterior ligament of the human TMJ. Oral Surg Oral Med Oral Pathol Oral Radiol Endod, 105(6), 698-701.

Ho, L. J., Lin, L. C., Hung, L. F., Wang, S. J., Lee, C. H., Chang, D. M., . . Tai, T. Y. (2005). Retinoic 
acid blocks pro-inflammatory cytokine-induced matrix metalloproteinase production by downregulating JNK-AP-1 signaling in human chondrocytes. Biochemical Pharmacology, 70(2), 200-208.

Jablonska-Trypuc, A., Matejczyk, M., \& Rosochacki, S. (2016). Matrix metalloproteinases (MMPs), the main extracellular matrix (ECM) enzymes in collagen degradation, as a target for anticancer drugs. J Enzyme Inhib Med Chem, 31(sup1), 177-183.

Jackson, M. T., Moradi, B., Smith, M. M., Jackson, C. J., \& Little, C. B. (2014). Activation of matrix metalloproteinases 2,9 , and 13 by activated protein $\mathrm{C}$ in human osteoarthritic cartilage chondrocytes. Arthritis Rheumatol, 66(6), 1525-1536.

Jiao, K., Zeng, G., Niu, L. N., Yang, H. X., Ren, G. T., Xu, X. Y., . . Wang, M. Q. (2016). Activation of alpha2A-adrenergic signal transduction in chondrocytes promotes degenerative remodelling of temporomandibular joint. Sci Rep, 6, 30085.

Jorgensen, A. E. M., Kjaer, M., \& Heinemeier, K. M. (2017). The Effect of Aging and Mechanical Loading on the Metabolism of Articular Cartilage. Journal of Rheumatology, 44(4), 410-417.

Kamiya, Y., Chen, J., Xu, M., Utreja, A., Choi, T., Drissi, H., \& Wadhwa, S. (2013). Increased mandibular condylar growth in mice with estrogen receptor beta deficiency. Journal of Bone and Mineral Research, 28(5), 1127-1134.

Kou, X. X., Wu, Y. W., Ding, Y., Hao, T., Bi, R. Y., Gan, Y. H., \& Ma, X. (2011). 17beta-estradiol aggravates temporomandibular joint inflammation through the NF-kappaB pathway in ovariectomized rats. Arthritis and Rheumatism, 63(7), 1888-1897.

Kufley, S., Scott, J. E., \& Ramirez-Yanez, G. (2017). The effect of the physical consistency of the diet on the bone quality of the mandibular condyle in rats. Archives of Oral Biology, 77, 23-26.

Kuroda, S., Tanimoto, K., Izawa, T., Fujihara, S., Koolstra, J. H., \& Tanaka, E. (2009). Biomechanical and biochemical characteristics of the mandibular condylar cartilage. Osteoarthritis and Cartilage, 17(11), 1408-1415.

Laaksonen, S., Nevalainen, T., Ketola, J., Hau, J., Nieminen, P., Haasio, K., . . Voipio, H.-M. (2017). Behaviour, stress and welfare of Sprague Dawley rats ( Rattus norvegicus ) on diet board feeding for 24 months. Applied Animal Behaviour Science, 194, 86-94.

Learreta, J. A., Bono, A. E., \& Durst, A. C. (2011). HLA analysis in patients with degenerative diseases of the temporomandibular joint. Cranio, 29(1), 32-37.

Liu, Y. D., Liao, L. F., Zhang, H. Y., Lu, L., Jiao, K., Zhang, M., . . Wang, M. Q. (2014). Reducing dietary loading decreases mouse temporomandibular joint degradation induced by anterior crossbite prosthesis. Osteoarthritis Cartilage, 22(2), 302-312.

Loeser, R. F. (2009). Aging and osteoarthritis: the role of chondrocyte senescence and aging changes in the cartilage matrix. Osteoarthritis and Cartilage, 17(8), 971-979.

Mähler, M., Berard, M., Feinstein, R., Gallagher, A., Illgen-Wilcke, B., Pritchett-Corning, K., \& Raspa, M. (2014). FELASA recommendations for the health monitoring of mouse, rat, hamster, guinea pig and rabbit colonies in breeding and experimental units. Laboratory Animals, 48(3), 178-192.

Manfredini, D., Guarda-Nardini, L., Winocur, E., Piccotti, F., Ahlberg, J., \& Lobbezoo, F. (2011). Research diagnostic criteria for temporomandibular disorders: a systematic review of axis I epidemiologic findings. Oral Surgery, Oral Medicine, Oral Pathology, Oral Radiology and Endodontics, 112(4), 453-462.

Mirahmadi, F., Koolstra, J. H., Lobbezoo, F., van Lenthe, G. H., \& Everts, V. (2017). Ex vivo thickness measurement of cartilage covering the temporomandibular joint. Journal of Biomechanics, 52, 165168. 
Murphy, G., Knauper, V., Atkinson, S., Butler, G., English, W., Hutton, M., . . Clark, I. (2002). Matrix metalloproteinases in arthritic disease. Arthritis Res, 4 Suppl 3, S39-49.

Nickel, J. C., McLachlan, K. R., \& Smith, D. M. (1988). A theoretical model of loading and eminence development of the postnatal human temporomandibular joint. J Dent Res, 67(6), 903-910.

Nielsen, R. H., Christiansen, C., Stolina, M., \& Karsdal, M. A. (2008). Oestrogen exhibits type II collagen protective effects and attenuates collagen-induced arthritis in rats. Clinical and Experimental Immunology, 152(1), 21-27.

Ohrbach, R., \& Dworkin, S. F. (2016). The Evolution of TMD Diagnosis: Past, Present, Future. Journal of Dental Research, 95(10), 1093-1101.

Orajarvi, M., Thesleff, I., Hartikainen, H., Raustia, A., \& Pirttiniemi, P. (2015). Effect of Estrogen and Food Hardness on Metabolism and Turnover of Condylar Cartilage. J Oral Facial Pain Headache, 29(3), 297-307.

Perez, C. V., de Leeuw, R., Okeson, J. P., Carlson, C. R., Li, H. F., Bush, H. M., \& Falace, D. A. (2013). The incidence and prevalence of temporomandibular disorders and posterior open bite in patients receiving mandibular advancement device therapy for obstructive sleep apnea. Sleep Breath, 17(1), 323-332.

Pirttiniemi, P., Kantomaa, T., \& Sorsa, T. (2004). Effect of decreased loading on the metabolic activity of the mandibular condylar cartilage in the rat. European Journal of Orthodontics, 26(1), 1-5.

Pirttiniemi, P., Kantomaa, T., \& Tuominen, M. (1993). Increased condylar growth after experimental relocation of the glenoid fossa. J Dent Res, 72(9), 1356-1359.

Rusu, M. C., Pop, F., Leonardi, R., Motoc, A. G., \& Jianu, A. M. (2011). Morphologic features of the fetal mandibular condyle: layers, canals and microvascular pattern. Ann Anat, 193(5), 436-446.

Schultz, G. S., \& Wysocki, A. (2009). Interactions between extracellular matrix and growth factors in wound healing. Wound Repair and Regeneration, 17(2), 153-162.

Sun, S., Bay-Jensen, A. C., Karsdal, M. A., Siebuhr, A. S., Zheng, Q., Maksymowych, W. P., . . . Henriksen, K. (2014). The active form of MMP-3 is a marker of synovial inflammation and cartilage turnover in inflammatory joint diseases. BMC Musculoskelet Disord, 15, 93.

Tanaka, E., Detamore, M. S., \& Mercuri, L. G. (2008). Degenerative disorders of the temporomandibular joint: etiology, diagnosis, and treatment. Journal of Dental Research, 87(4), 296-307.

Tiilikainen, P., Pirttiniemi, P., Kainulainen, T., Pernu, H., \& Raustia, A. (2005a). MMP-3 and -8 expression is found in the condylar surface of temporomandibular joints with internal derangement. J Oral Pathol Med, 34(1), 39-45.

Tiilikainen, P., Pirttiniemi, P., Kainulainen, T., Pernu, H., \& Raustia, A. (2005b). MMP-3 and -8 expression is found in the condylar surface of temporomandibular joints with internal derangement. Journal of Oral Pathology and Medicine, 34(1), 39-45.

Traub, R. J., \& Ji, Y. (2013). Sex differences and hormonal modulation of deep tissue pain. Frontiers in Neuroendocrinology, 34(4), 350-366.

van Meurs, J., van Lent, P., Stoop, R., Holthuysen, A., Singer, I., Bayne, E., . . van den Berg, W. (1999). Cleavage of aggrecan at the Asn341-Phe342 site coincides with the initiation of collagen damage in murine antigen-induced arthritis: a pivotal role for stromelysin 1 in matrix metalloproteinase activity. Arthritis and Rheumatism, 42(10), 2074-2084.

Wang, X. D., Zhang, J. N., Gan, Y. H., \& Zhou, Y. H. (2015). Current understanding of pathogenesis and treatment of TMJ osteoarthritis. Journal of Dental Research, 94(5), 666-673.

Warren, M. P., \& Fried, J. L. (2001). Temporomandibular disorders and hormones in women. Cells 
Tissues Organs, 169(3), 187-192.

Williams, R. O., Feldmann, M., \& Maini, R. N. (2000). Cartilage destruction and bone erosion in arthritis: the role of tumour necrosis factor alpha. Annals of the Rheumatic Diseases, 59 Suppl 1, i75-80.

Ye, T., Sun, D., Mu, T., Chu, Y., Miao, H., Zhang, M., . . Y Yu, S. (2018). Differential effects of highphysiological oestrogen on the degeneration of mandibular condylar cartilage and subchondral bone. Bone, 111, 9-22. 
Table 1. Distribution of Experimental Animals

\begin{tabular}{ccccc}
\hline \multirow{2}{*}{ Age } & Estrogen & \multicolumn{3}{c}{ Dietary loading } \\
\cline { 3 - 5 } & & Powder & Pellet & Diet board \\
\hline \multirow{2}{*}{ 5-month-old } & OVX & 8 & 8 & 8 \\
\cline { 2 - 5 } 14-month-old & non-OVX & 8 & 8 & 8 \\
& OVX & 8 & 8 & 8 \\
& non-OVX & 6 & 8 & 7 \\
\hline
\end{tabular}

Table 2. $P$ values of type III tests* of fixed effects of dietary loading, estrogen level, and aging

\begin{tabular}{cccccc}
\hline \multirow{2}{*}{$\begin{array}{c}\text { Thickness of } \\
\text { anterior part }\end{array}$} & Thickness of & Thickness of & MMP-3 & MMP-8 \\
& central part & posterior part & 0.035 & 0.538 \\
Dietary loading & $<0.001$ & $<0.001$ & 0.912 & 0.855 & $<0.001$ \\
Estrogen level & 0.991 & 0.364 & 0.644 & 0.204 & $<0.001$ \\
\hline
\end{tabular}

* The type III tests of fixed effect with linear mixed model

Dependent variables: thickness, MMP-3 and MMP-8

Independent variables: dietary loading, estrogen level and aging adjusted with the two-way interaction terms 


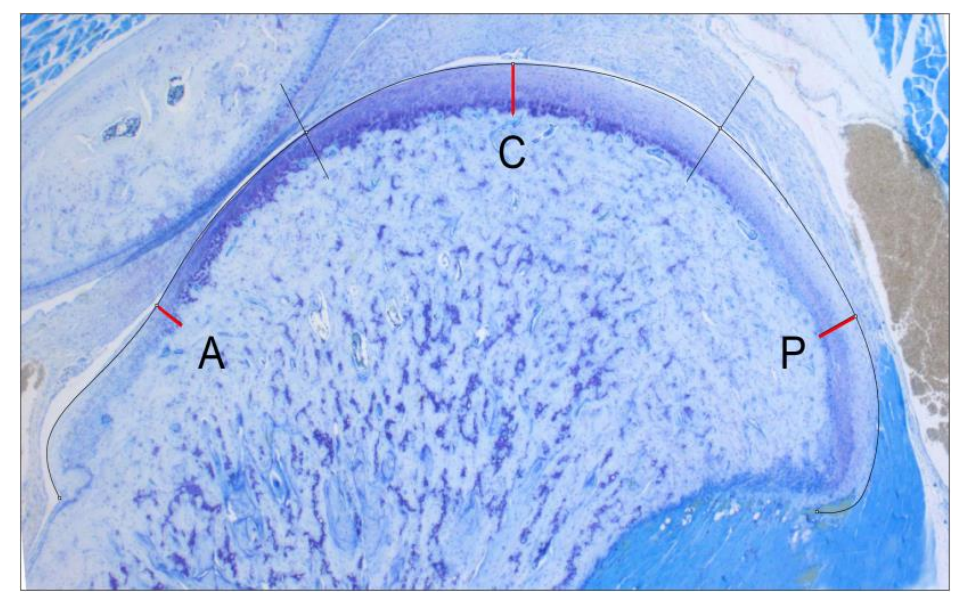

Figure 1. The mandibular condylar cartilage $(\mathrm{MCC})$ was divided sagittally into three equal parts according to the articular surface: $\mathrm{A}=$ anterior part, $\mathrm{C}=$ most central part, $\mathrm{P}=$ posterior part. Red line represents the midpoint of anterior, central and posterior part. The condylar image was taken from a 14month-old OVX rat on the diet board. 


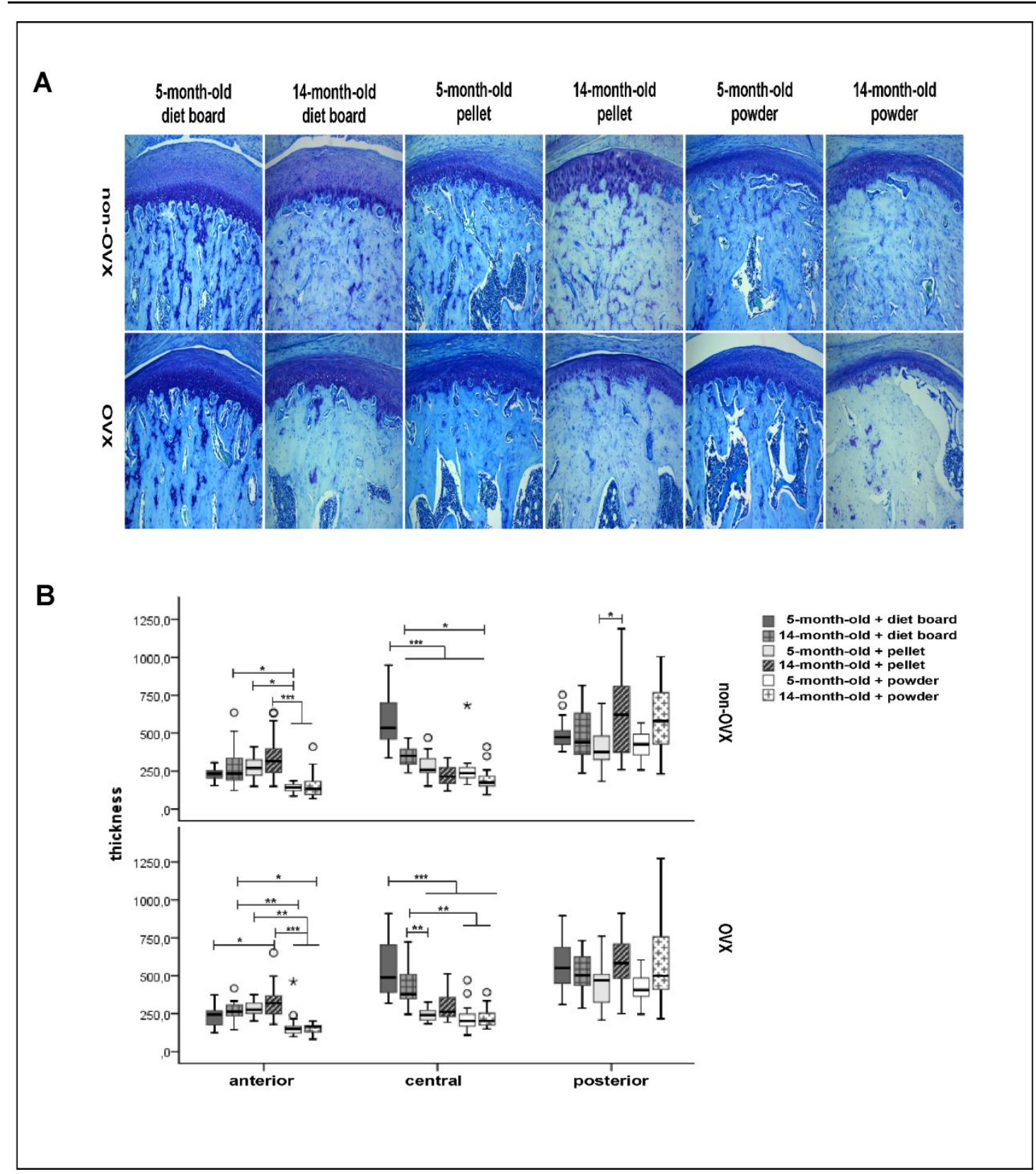

Figure 2. Changes of the thickness (A) Toluidine blue staining from the central part of the mandibular condylar cartilage (MCC). Scale bar, $200 \mu \mathrm{m}$. (B) Box plot diagram showing the thickness of the three parts. ${ }^{*} P<0.05$. ${ }^{* *} P<0.01$. ${ }^{* * *} P<0.001$. - shows difference including all the groups under the line. 


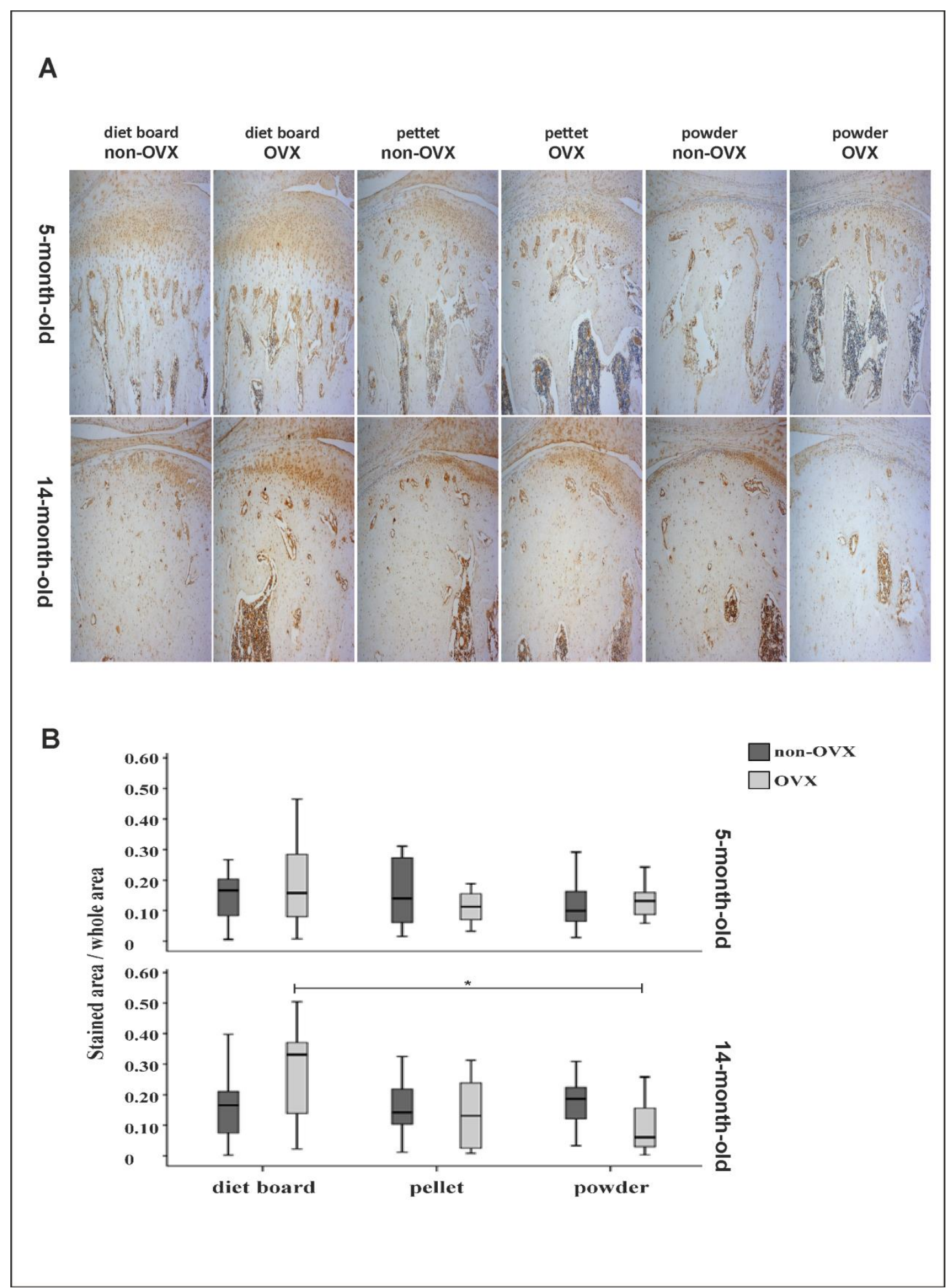

Figure 3. Expression of MMP-3 in the mandibular condylar cartilage (MCC). (A) Immunohistochemical staining showing the central part of the condyle. Scale bar, $200 \mu \mathrm{m}$. (B) Box plot diagram illustrating MMP-3 expression. ${ }^{*} P<0.05$. 


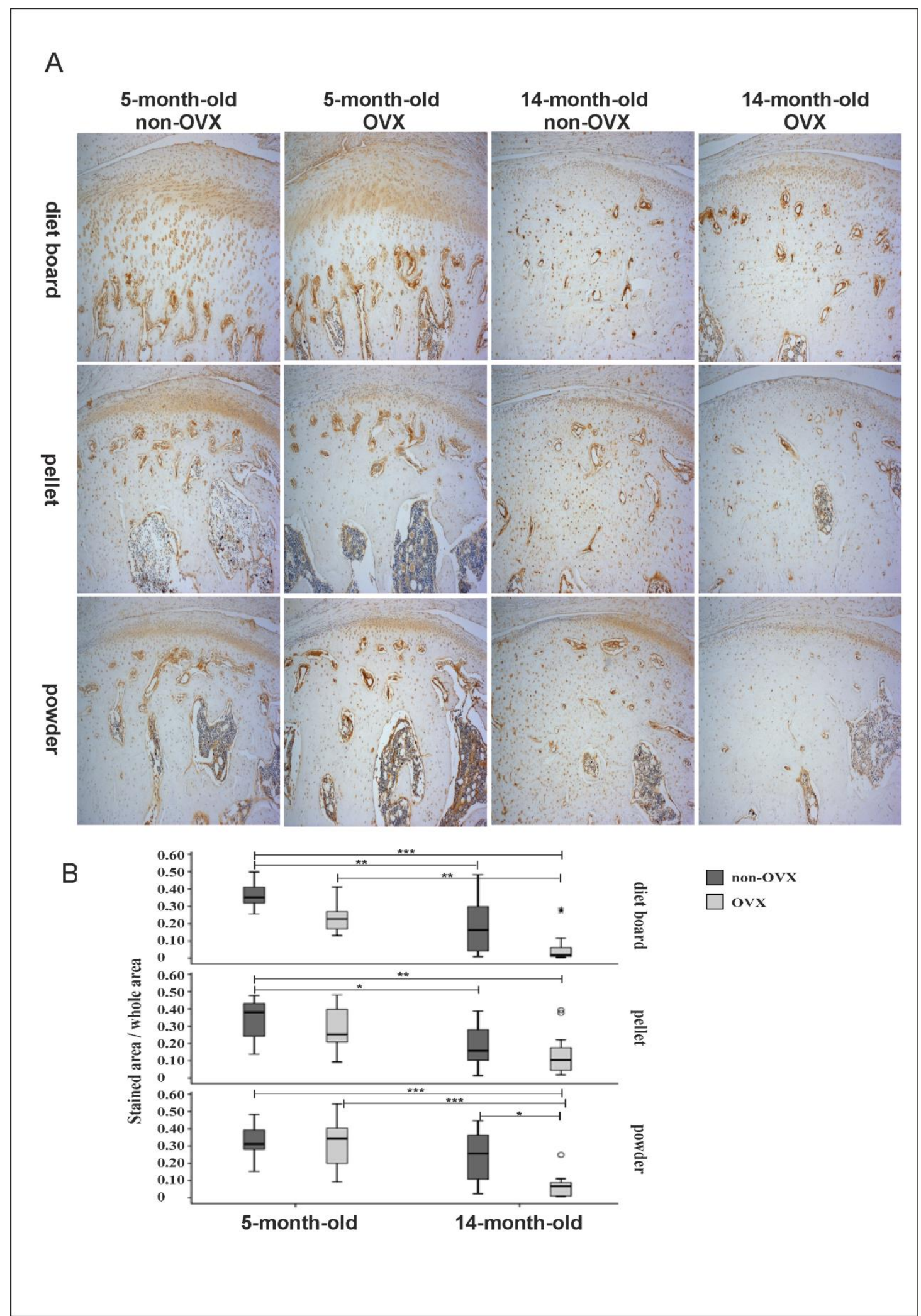

Figure 4. Expression of MMP-8 in the mandibular condylar cartilage (MCC). (A) Immunohistochemical staining showing the central part of the condyle. Scale bar, $200 \mu \mathrm{m}$. (B) Box plot diagram illustrating MMP-8 expression. ${ }^{*} P<0.05$. ${ }^{* *} P<0.01 .{ }^{* * *} P<0.001$. 